\title{
The Roughness of Crystal Surfaces, a Clarified Problem
}

\author{
S. Balibar, Paris
}

(Groupe de Physique des Solides de l'Ecole Normale Supérieure)

Not only today is the physics of crystal surfaces one of the very large research fields, but it has been so since many decades. It comprises a wide variety of problems which belong to statistical physics, solid state physics, metallurgy, physico-chemistry, physics of non-linear phenomena, materials science, technology, etc. One might thus naïvely believe that one has known for a very long time why, at a given temperature, the surface of a crystal forms a facet or not, more precisely, whether it is in a "smooth" or "rough" state. How drastically roughness governs crystal growth could also be thought to be a simple problem which was solved long ago.

Answering these questions however, supposes that one understands what the equilibrium structure of a crystal surface is. In my opinion, this was not so clear until very recently, although the problem is now solved, thanks to difficult renormalization calculations ${ }^{1,2}$ ) and careful experiments in helium- ${ }^{3,4}$ ). These theoretical and experimental studies have been done during the last decade and have given consistent and definite results only this year. In particular, the way in which a surface goes from rough to smooth or vice versa, when its temperature is changed, is now well understood. The phenomenon is known as the "roughening transition" and will be the central point of this article.

\section{Why Helium-4?}

At a certain temperature which depends on the ambient pressure, a pure system may present two phases in thermodynamic equilibrium, for example a crystal and a fluid. The fluid may be a vapour or a liquid, but the distinction has not the slightest importance for our purpose. We want to consider the properties of the boundary between the two phases, which are supposed at or close to equilibrium. It is a more or less twodimensional surface which is stable, of course, when complete thermodynamic equilibrium is achieved. Only then has the crystal its equilibrium shape. What does this precisely mean?

The temperature must be homogeneous in the whole system, and the pressure such that the liquid and solid chemical potentials are also the same everywhere. In practice, slight deviations from the exact melting pressure exist; they are due to the effect of gravity or local curvature of the interface and are crucial to the determination of equilibrium crystal shapes. A close analogy, which we all know from everyday experience, is the equilibrium shape of the surface of, say, water in a glass: on the liquid side, the pressure is constant over all flat horizontal parts but differs slightly at the edges which are bent as a result of wetting and capillary effects. Similarly for crystals, but true equilibrium with their liquids is usually very difficult to observe: the slightest temperature deviation in the system has drastic effects on the crystal shape. Herein lies the main advantage of working with helium-4. Below about $2 \mathrm{~K}$, the liquid is a superfluid and its heat conductivity may be considered as infinite. Moreover, the crystal is of such a high quality that it may be an even better thermal conductor. In addition, the melting latent heat is very small, the liquid viscosity is nearly zero and the impurity concentration easy to lower below $10^{-9}$. As a consequence, in helium-4, true equilibrium shapes may be obtained in times ranging from a millisecond to a minute over which thermodynamic parameters (i.e. pressure and temperature) are accurately controllable. The properties of liquidsolid interfaces are thus accessible in simple experiments, whereas in classical systems, enormous response times, due to the slow diffusion of heat mass or impurity concentration, make the interface properties much more difficult to study; in most situations, they are hidden by bulk properties.

The very short response times in helium-4 make it possible to realise experiments which are as common with liquid surfaces as they are rare with ordinary crystal surfaces. The most striking example is on the propagation of crystallization waves which was first performed by K.O. Keshishev ${ }^{5}$ ) in 1979. It illustrates rather well how fast helium-4 crystals may respond to external perturbations. These waves are the exact analogue of capillary waves on a free liquid surface which result from the combination of a restoring force - the surface tension - and the inertia of the liquid.
Both have the same kind of dispersion relation, except that for water, the attenuation comes from the liquid viscosity whereas for helium it reflects the finite growth rate responding to a finite perturbation. Crystallization waves result from local growth and melting; no motion in the solid is involved. In classical systems, growth rates are much too small and the waves are overdamped. But at low enough temperature $(T \leq 0.4$ $\mathrm{K})$ and on "rough" helium surfaces, crystallization waves propagate as readily as

Fig. 1 - Helium-4 crystals are completely rounded, or "rough" in all directions, above $1.3 \mathrm{~K}(\mathrm{a})$. Three roughening transitions have been observed at lower temperature. They respectively concern (0001), (1100) and (1T 01) surfaces which appear as facets below $T_{R 1}=1.28 \mathrm{~K}, T_{R 2} \cong 1 \mathrm{~K}$ and $T_{R 3} \cong$ $0.35 \mathrm{~K}$. These facets are visible on the following photographs which respectively show crystals growing slowly at $1.08 \mathrm{~K}(\mathrm{~b})$, $0.4 K(c)$ and $0.35 K(d)$.

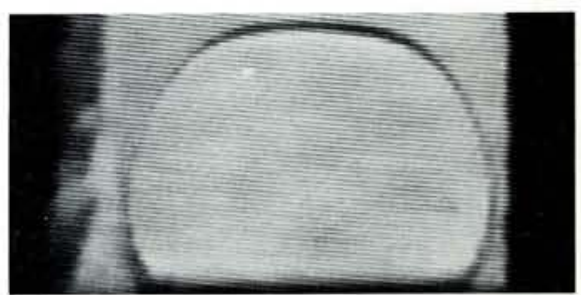

(a)

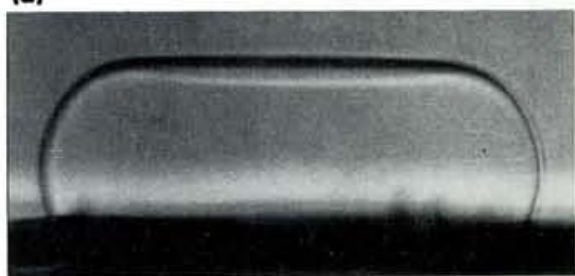

(b)

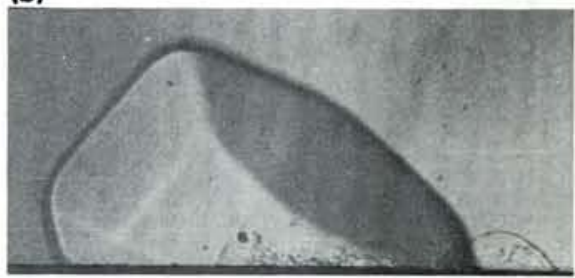

(c)

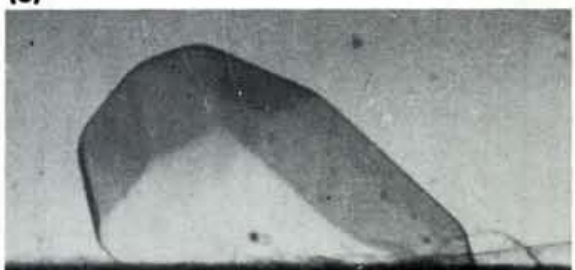

(d) 
ripples on water. They are easy to see in an optical cryostat: when shaking it slightly, the crystal surface moves as would water in a glass, while the bulk crystal, perfectly steady, keeps its very good quality.

Once this has been understood, it is easier perhaps to appreciate that one may study the properties of helium crystal surfaces in all sorts of capillary experiments. For example, as will be detailed below, one may blow up crystalline bubbles under known pressure differences and measure their curvature, or run communicating vessel experiments. Such experiments have shown that growth shape drastically depends on temperature: above $1.3 \mathrm{~K}$ crystals look rounded everywhere but, when cooled down, three successive kinds of facet appear on their surface (Fig. 1). These changes are "roughening transitions" and have been studied in detail recently.

\section{Rough or Smooth}

What are the differences between a crystal surface and a simple liquid surface? The main difference stems from the crystal being a system which is periodic in space. A liquid surface is free to occupy any position whereas a crystal surface may be restricted. Let us first neglect thermal fluctuations and consider a crystal near absolute zero with its high symmetry axis vertical. One may guess that the equilibrium crystal surface is more or less the last horizontal crystal plane. Also the crystal should grow slowly, layer by layer, from the lateral motion of well defined steps of atomic height. This says nothing other than that the crystal surface is localized or anchored to the lattice. Also if one is to write a surface hamiltonian, it should contain a periodic potential, meaning that equally spaced positions of the surface have minimal energies. We have just described what is called a smooth surface and it is not pure imagination since it closely corresponds to all the beautiful symmetrical facets one can see on many natural crystals.

Now what happens if one raises the temperature? Thermal fluctuations become important; more and more atoms continuously arrive on the surface or leave it. As a consequence the surface is invaded by defects like adatoms (or inversely advacancies), terraces of various sizes and increasing thickness, steps in all directions, etc. Very nice computer simulations ${ }^{6}$ ) have shown this (Fig. 2) and one realises that the interface starts wandering with respect to the lattice. This is like a particle moving in a periodic series of potential wells: at
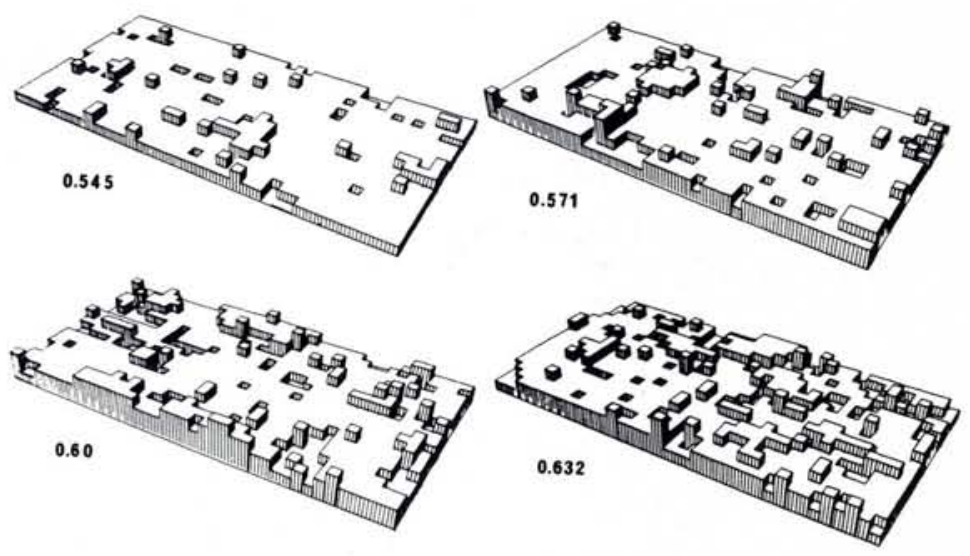

Fig. 2 - As illustrated by these numerical simulations ${ }^{6}$ ), the microscopic aspect of a crystal surface depends on temperature (here appearing as a number in reduced units). At $T=0.545<T_{R}$ the surface is smooth with a strictly positive step energy; it is anchored on one of the lattice planes, with few thermally activated defects (adatoms, terraces, steps). On the contrary, at $T$ $=0.667>T_{R}$, the surface is rough, invaded by steps whose energy has vanished, becoming delocalized with respect to the lattice.

low temperature, the particle stays in one of them, at higher temperature it may jump to neighbouring wells and become mobile, delocalized. A striking aspect is that a crystal surface with fluctuations large enough to move over many lattice spacings does not feel this lattice any more: the fluctuations average the effect of the lattice potential to zero and the crystal surface becomes exactly as free as a fluid surface. It thus seems that on warming a crystal, its surface should progressively change and become very disordered, full of defects, delocalized, or simply "rough" as everybody now says. In their historical paper ${ }^{7}$ ), Burton Cabrera and Frank proposed the existence of these two possible states for crystal surfaces; this was 35 years ago. But the problem was to know whether a real phase transition occurs between the smooth and the rough state, and what could be its nature (how physical quantities vary close to it). In my opinion, the solution to this problem has progressively emerged over this last decade. Various theoretical approaches have been used but lead to the same qualitative predictions which I want to summarize now.

The roughening transition exists. It takes place at a certain temperature $T_{R^{\prime}}$ but it is one of the most continuous phenomena in Nature. Indeed, no thermodynamic quantity like the surface energy has a discontinuity, nor any of its temperature derivatives. In particular, the free energy of a step vanishes exponentially at $T_{R}$. This quantity describes the ener-

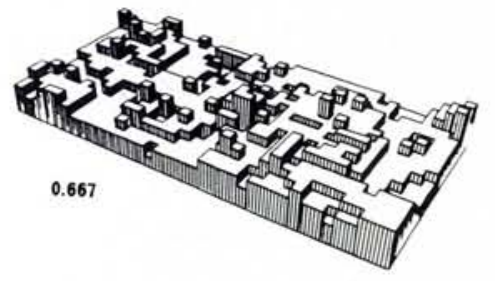

gy per unit length of the boundary between some region of the surface and an adjacent region whose height differs by one lattice spacing. Since this step energy $E_{\mathrm{S}}$ is found to be zero in the rough (disordered) state and strictly positive in the smooth (ordered) state, it is often presented as an order parameter for the surface. One may understand that if $E_{\mathrm{S}}$ is finite there are very few steps and the surface is nearly a (smooth) lattice plane, whereas, if it is zero, the (rough) surface is invaded by zero energy defects everywhere.

The various theoretical approaches also predict what should happen with the growth rate and the surface stiffness around $T_{\mathrm{R}}$. In the rough state, growth proceeds via the sticking of individual atoms on the numerous surface sites. It is a fast process which is linear in the sense that the growth velocity is proportional to the departure from equilibrium, or driving force on the surface, i.e. the chemical potential difference $\Delta \mu$ between the liquid and the solid. On the contrary, in the smooth state, and in the absence of dislocations, the growth has to proceed via the nucleation of two dimensional terraces which have a finite energy. As a result, the growth rate is small and depends exponentially on the driving force. The exact way in which the growth rate changes through the roughening transition is subtle, but becomes clear when we examine the experimental results.

As to the surface stiffness, it is the anisotropic quantity which governs the crystal curvature, the equations being a generalization of Laplace's law which relates the liquid surface tension to the pressure difference across the bent liquid surface. Theorists have found ${ }^{8,9}$ ) 


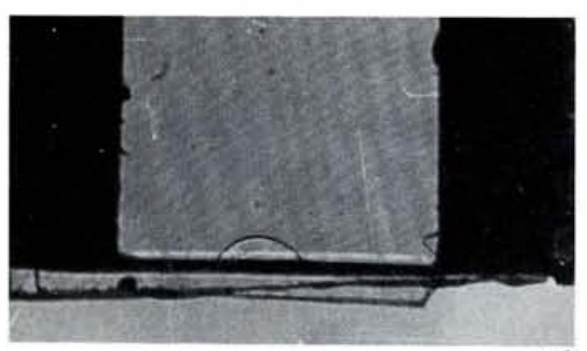

a)

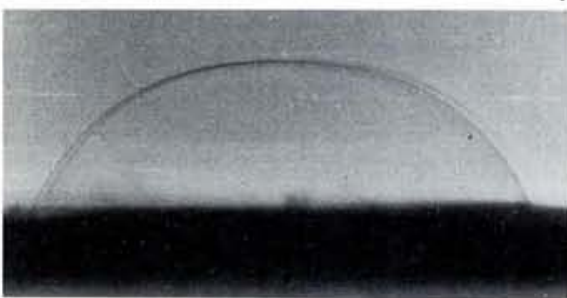

b)

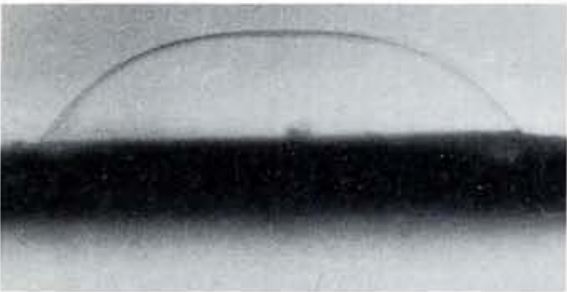

c)

Fig. 3 - These three photographs illustrate the particular method used by Wolf et al. ${ }^{3)}$ to measure the surface stiffness of $\mathrm{He}-4$ crystals.

a) A transparent box is immersed in the crystal and has a small hole $(\varnothing=0.8 \mathrm{~mm})$ drilled in its bottom plate. Outside the box, the liquid-solid interface (dark horizontal line) lies about $5 \mathrm{~mm}$ above the bottom and touches the vertical walls with an angle of about $140^{\circ}$. Inside the box, a meniscus is anchored on the hole edges. Its visible profile is bent as a result of a finite pressure difference between the solid below and the liquid above. Due to hydrostatic equilibrium in the liquid and thermodynamic equilibrium at the interface, this pressure difference is simply related to the height difference with res pect to the outside. The surface stiffness is obtained from a generalized Laplace law.

b) Close to the roughening temperature $(1.28 \mathrm{~K})$ the enlarged meniscus looks rounded everywhere with a finite curvature on top as predicted by Jayaprakash Saam and Teitel 8).

c) At $1.13 K$, the slight flattening of the top indicates that one is far enough below the roughening transition for a macroscopic facet to exist on the crystal surface. At even lower temperature, menisci look similar to Fig. $1 b$.

that the surface stiffness should have a rather peculiar behaviour close to $T_{R}$ : it should be infinite in the smooth phase, which means that even a large pressure difference between the liquid and the solid is unable to bend a facet. On the contrary, a rough surface may be bent, and it generally appears rounded on equilibrium crystal shapes. Its surface stiffness is finite and decreases slowly as the square root of temperature from a universal value $\gamma^{*}$ at $T_{\mathrm{R}}$. This is an important prediction which is expressed mathematically by the simple equation:

$$
k_{\mathrm{B}} T_{\mathrm{R}}=(2 / \pi) \gamma^{*} d^{2}
$$

where $k_{\mathrm{B}}$ is Boltzmann's constant and $d$ the minimum distance between equivalent crystal planes parallel to the considered surface. This remarkable result is called "universal" because it does not explicitly depend on any microscopic detail of the surface. Moreover I should like to emphasize that this universal relation is the modern roughening criterion. For a given crystal, if one knows the surface stiffness $\gamma$, one can predict the set of roughening temperatures at which facets appear or disappear on the surface. They are related to the various possible values of $d$; unless $\gamma$ is very anisotropic, the most stable facets are the densest crystal planes which have the largest spacings $d$. At lower and lower temperatures, more and more facets appear which have lower symmetries and smaller surface densities.

\section{Experimental Confirmations}

Returning to the helium -4 crystals, we have already made allusion to one way of determining their surface stiffness (Fig. 3), by measuring the curvature of surfaces across which a known pressure difference is applied. Using this technique Wolf et al. ${ }^{3}$ ) derived the surface stiffness $\gamma$ of helium crystals around the (0001) direction which presents a roughening transition at $1.28 \mathrm{~K}$. This direction is the six-fold symmetry axis of the hexagonal close packed structure. They found a value which was close to the universal prediction. A slight apparent discrepancy could be attributed to the need to calculate the second derivative of the crystal profile averaged over a small angular window since they could not obtain $\gamma$ strictly in the (0001) direction. Careful accounting for such angular effects finally produced very good agreement with the theoretical predictions. Agreement was also found for roughening transitions concerning other facets, at least the $(10 \overline{10})$ around 1 $\mathrm{K}$. The universal relation or roughening criterion thus seems valid.

However, more precise quantitative confirmation has come, in my opinion, from measurements of growth rates. Wolf et al. ${ }^{3}$ ), then Gallet et al. ${ }^{4}$ ) with more accuracy, looked at the relaxation of a crystal surface in a communicating vessels experiment. In a small region of their experimental cell (a small transparent box), the crystal surface was prepared at a lower level than everywhere else. Then its height was measured with an accuracy of one micron by an interferometric technique during relaxation towards equilibrium (velocities ranged from 1-100 $\mu \mathrm{m} / \mathrm{s})$. The difference in chemical potential $\Delta \mu$ between the liquid and the solid, was simply proportional to the height difference whose time derivative gave the growth rate $V$. The important goal was to measure the growth characteristics $V(\Delta \mu)$ and to analyze its temperature evolution around $T_{\mathrm{R}}$. Complete and precise quantitative agreement was found with the theory as very recently improved by $P$. Nozières et al. ${ }^{2}$ ).

To summarize, in the smooth state ( $T$ $<T_{R}$ ) a facet grows by thermal activation of two dimensional terraces. The terraces themselves have a minimal (or "critical") size to start growing and their free energy depends drastically on the step energy, because their boundary is such a step. This leads to a growth rate which varies exponentially with both the step energy and the departure from equilibrium $\Delta \mu$. Wolf and Gallet brought evidence for this which in itself is interesting; even though such a growth process is widely accepted to exist, its quantitative analysis has rarely been achieved. But they mainly used their data to obtain the temperature variation of the step free energy, i.e. the critical behaviour of the order parameter close to the roughening transition (here that of (0001) facets at $1.28 \mathrm{~K}$ ). This behaviour was found to be the very continuous exponential one had predicted a few years before (Fig. 4a). One should also mention that whilst a few other theories, "mean field" theories in particular have been published, they did not correctly account for the fluctuations in the system and are all ruled out by these recent experiments.

Finally, very close to $T_{R}\left(T \leqq T_{R}\right)$, an intermediate regime exists which corresponds to the situation where the surface is not completely rough, but the fluctuations have a larger amplitude than the critical size of the terraces involved in the growth. This is the temperature domain where the growth rate increases from very small (in the smooth state) to large (in the rough state) (Fig. 4b). As explained by Gallet et al., the temperature width of this intermediate growth regime increases with the applied difference in chemical potential: it is a dynamic broadening of the roughening transition which is fully and quantitatively explained by the theory. Indeed, after numerical integration of the renormalization equations obtained by Nozières, Gallet et al. showed that all their measurements (curvature, step 


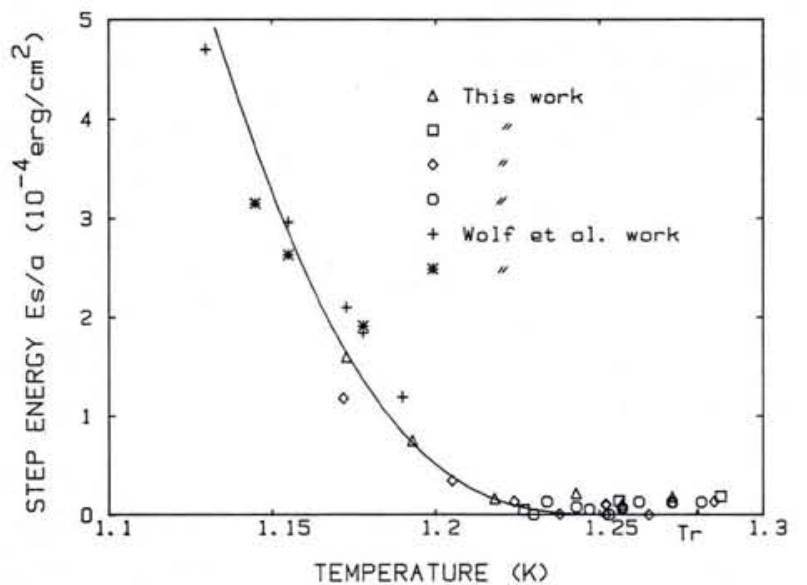

Fig. 4 - (Left) The critical theory of roughening (solid lines) predicts ") that the step energy on a facet vanishes exponentially when $T$ approaches the roughening temperature $T_{R}$. (Here $T_{R}=1.28 \mathrm{~K}$ for (0001) $\mathrm{fa}$ cets on a hcp He-4 crystal).

(Right) It also predicts 2) that the growth rate should increase from nearly zero to a finite value at $T_{R}$ in a temperature domain whose width depends on the exact departure from equilibrium (here a height difference H). Good agreement was found by Gallet et al. 4) with their experiments (various symbols).

energies, growth rates for three different departures from equilibrium) could be very well described with a single choice of the three parameters involved: the roughening temperature $T_{R}$, the magnitude of the coupling of the crystal surface to the underlying lattice, and the short wavelength cut-off of the fluctuations la sort of zero temperature thickness for the surface). Theory and experiments have in my opinion now reached such a degree of precision and agreement that the problem of crystal roughness looks solved, at least for simple surfaces.

\section{Vicinal Surfaces and Dendrites}

I have focussed above on one single aspect, but many other experiments on helium crystals have been done recently $\left.{ }^{10}\right)$. In particular, the way in which heat goes through their surface has also been analyzed. This too is a phenomenon which depends drastically on the roughness and I can see at least two other directions in which important progress could be made in the near future.

The first one concerns vicinal surfaces. A vicinal surface is a crystal surface which is slightly tilted with respect to one of the main crystal planes. As a consequence, at a low enough temperature, it is made out of a series of parallel steps whose interactions are not so well known. The roughness of such vicinal surfaces has been studied recently on

Name:

Address:

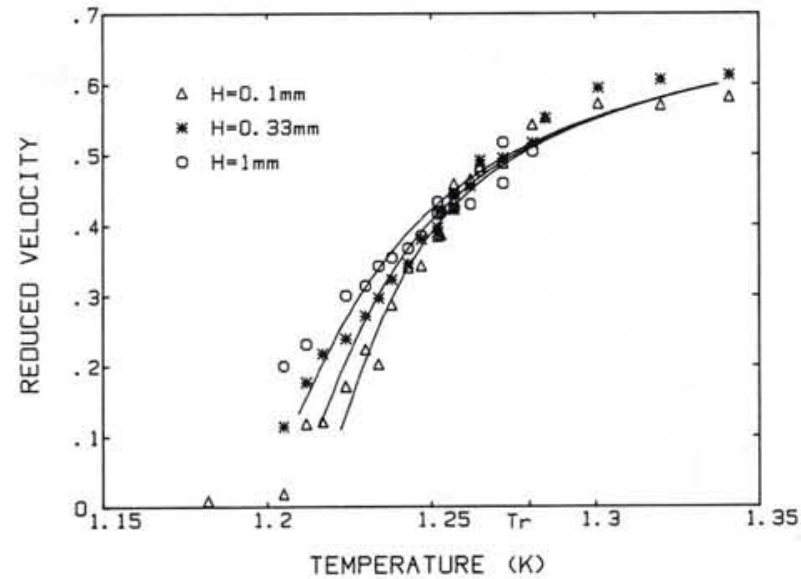

NOW AVAILABLE!

MATERIALS SCIENCE REPORTS

\section{A REVIEW JOURNAL}

This new review journal will serve the Materials Science community in two ways. It will provide a general background of materials science and present specialized reviews on current and significant developments in the field. Invited review papers will cover a broad spectrum of materials science encompassing materials systems such as electronic and nuclear materials, ceramics, polymers and metals.

Emphasis will be placed on materials properties (e.g. physical, chemical, electrical and mechanical), methods of preparation, constituent interactions as well as techniques for characterization and modification. Both theoretical and experimental papers will be featured.

Coordinating Editors: S.S. Lau, San Diego, CA, USA; F.W. Saris, Amsterdam, The Netherlands.

Editorial Board: W. Bauer, Livermore, CA, USA; A.G. Cullis, Great Malvern, UK; S. Furukawa, Yokohama, Japan; E. Kaldis, Zürich, Switzerland; E. Kramer, Ithaca, NY, USA; J.W. Mayer, Ithaca, NY, USA; T. Tokuyama, Tokyo, Japan; K.N. Tu, Yorktown Heights, NY, USA.

The first issue contains the review: NEW PERMANENT MAGNET MATERIALS by K.H.J. Buschow

The following reviews will appear in future issues:

N.W. Cheung: Megavolt Ion Implantation in Semiconductors and their Interfaces; L. Csepregi: Micromechanics: Silicon Microfabrication Technology: P.J. Grunthaner, M.H. Hecht, F.J. Grunthaner: Photoemission Studies of the SiO2Si Interface; T.F. Kuech: Metal-Organic Vapor Phase Epitaxy of Compound Semiconductors; L.G. Meiners, H.H. Wieder: Semiconductor Passivation; G.L. OIson, J.A. Roth: Kinetics and Mechanisms of Solid Phase Crystallization in Amorphous Silicon; P.S. Peercy, M.O. Thompson: Measurement of Melt and Solidification Dynamics; K. Gamo, R. Shimizu, S. Namba: Ion Beam Lithography.

Subscription Information:

1987: Volume 2 in 8 issues. Dfl.275.00/US $\$ 110.75$

(Subscribers to volume 2 will automatically receive volume 1 free of charge, during 1987). Price includes postage and handling costs. The dutch guilder price is definitive. Customers in the Netherlands, please add 5\% BTW.

\section{ASK FOR A FREE INSPECTION COPY!}

Please mail this coupon, or a photo-copy to:

North-Holland Physics Publishing,

attn. Ms. A. Broekgaarden, P.O. Box 103, 1000 AC Amsterdam,

The Netherlands

Customers in the USA and Canada, mail to:

Elsevier Science Publishing Co., Inc., 52 Vanderbilt Avenue, New York, N.Y. 10017

Please send me a free inspection copy of:

MATERIALS SCIENCE REPORTS 
crystals of $\mathrm{Cu}^{11}$ ), $\mathrm{Ni}^{12}$ ) and $\mathrm{Pb}{ }^{13}$ ). Their exact structure is, however, not yet as clear as it could be.

Another interesting direction is the influence of roughness on the dendritic instability. The diffusion of heat or impurity concentration being slow during growth, most growing crystals do not keep a stationary convex shape but develop one dimensional arms with lateral branches. Snowflakes are such dendritic patterns and steels are full of entangled dendrites. The theoretical treatment of this pattern selection problem is very difficult but it seems nowadays, that the crystal surface anisotropy plays an important role $\left.{ }^{14}\right)$. Since a roughening transition is a drastic change in the anisotropy of the crystal (facets appear in a given number of symmetric directions), it should affect the dendritic instability. This is precisely what was recently shown by E. Rolley et al. ${ }^{15}$ ) who studied helium- 3 crystals. The light isotope of helium is not superfluid except at extremely low temperature so that its growth is very different from that of helium-4. Dendrites can be observed (Fig. 5) which are killed and replaced by dodecahedra when the first roughening transition occurs (around $0.08 \mathrm{~K}$ ). To make this quantitative is much more difficult than with helium- 4 crystals, but I believe that the study of helium-3, after that of helium-4, can also improve our understanding of crystal surfaces and crystal growth.

\section{REFERENCES}

1. Weeks J.D. and Gilmer G.H., Adv. in Chem. Phys. 40 (1979) 157.

2. Nozières P. and Gallet F., to appear in J. Physique (1987).

3. WolfP.E., Gallet F., Balibar S., Rolley E. and Nozières P., J. Physique 46 (1985) 1987.

4. Gallet F., Balibar S. and Rolley E., to appear in J. Physique (1987).

5. Keshishev K.O., Parshin A.Ya. and Babkin A.B., Sov. Phys. JETP 53 (1981) 362.

6. Leamy H.J., Gilmer G.H. and Jackson K.A., Surface Physics of Materials 1, ed. J.D. Blakely (Academic Press, New York) 1975.

7. Burton W.K., Cabrera N. and Frank F.C., Phil. Trans. Roy. Soc. 243A (1951) 299.

8. Jayaprakash C., Saam W.F. and Teitel S., Phys. Rev. Lett. 50 (1983) 2017.

9. Fisher D.S. and Weeks J.D., Phys. Rev. Lett. 50 (1983) 1077.

10. Balibar S. and Castaing B., Surf. Science Rep. 5 (1985) 87.

11. Villain J., Grempel D.R. and Lapujoulade J., J. Phys. F 15 (1985) 809.

12. Conrad E.H., Aten R.M., Kaufman D.S., Allen L.R., Engel T. den Nijs M. and Riedel E.K., J. Chem. Phys. 84 (1986) 1015.

13. Heyraud J.C. and Metois J.J. submitted to Crystal Growth (1986).

14. Ben Jacob E., Goldenfeld N., Langer J.S. and Schön G., Phys. Rev. A 29 (1984) 330. 15. Rolley E., Balibar S. and Gallet F., Europhys. Lett. 2 (1986) 247.

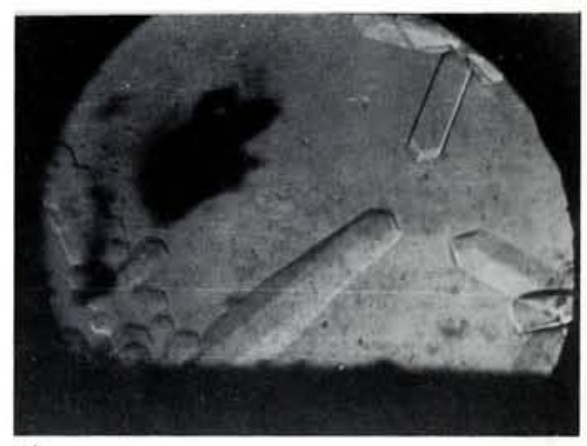

a)

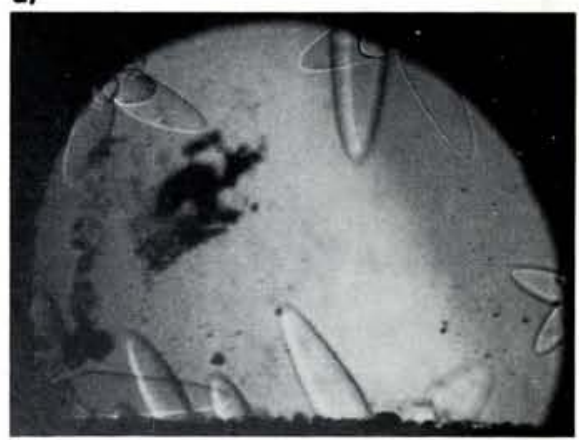

b)

Fig. 5. - a) Below the roughening temperature $T_{R} \cong 0.08 \mathrm{~K}$ of (110) facets, the anisotropy of the growth rate is so large that the growth shapes of helium-3 crystals are stable dodecahedra (here $T=0.07 \mathrm{~K}$ ).

b) Due to the slow diffusion of He-4 impurities (200 ppm in this sample), the growth of He-3 crystals above their roughening temperature is usually dendritic. These needle crystals were grown at $0.10 \mathrm{~K}$.

\section{Z11 zолен}

Applications are invited for a faculty position in PHYSICAL ELECTRONICS at the Swiss Federal Institute of Technology in Zurich.

The new professor will be responsible for teaching sensor and materials technology and its applications at undergraduate and graduate levels. Possible areas of research include materials development, novel devices and sensors related to applications in microelectronics, robotics and communications technology.

The successful applicant will have several years of experience in research in the above mentioned fields, either in basic science or applications, and from solid state and semiconductor physics to bioelectronics. Proven ability to perform and direct advanced research and the willingness to cooperate and to teach at all university levels are expected. A working knowledge of German is required.

Applications with curriculum vitae and list of publications, patents, etc. should be sent before 31 December, 1986, to the President of the ETH Zurich, Prof. H. Ursprung,

ETH-Zentrum, CH-8092 Zurich.

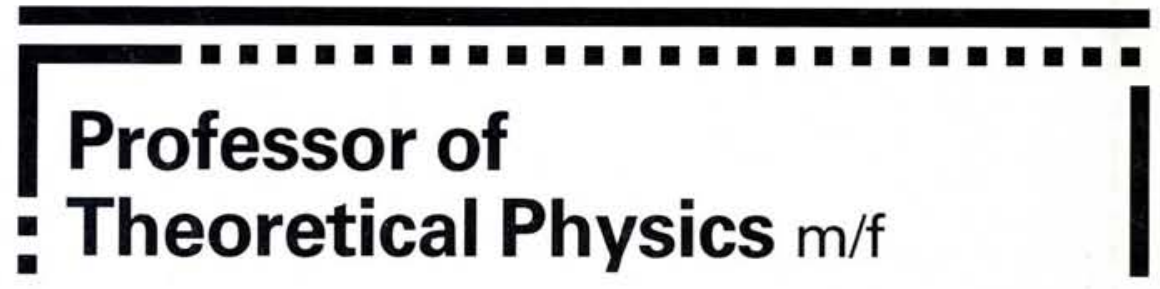

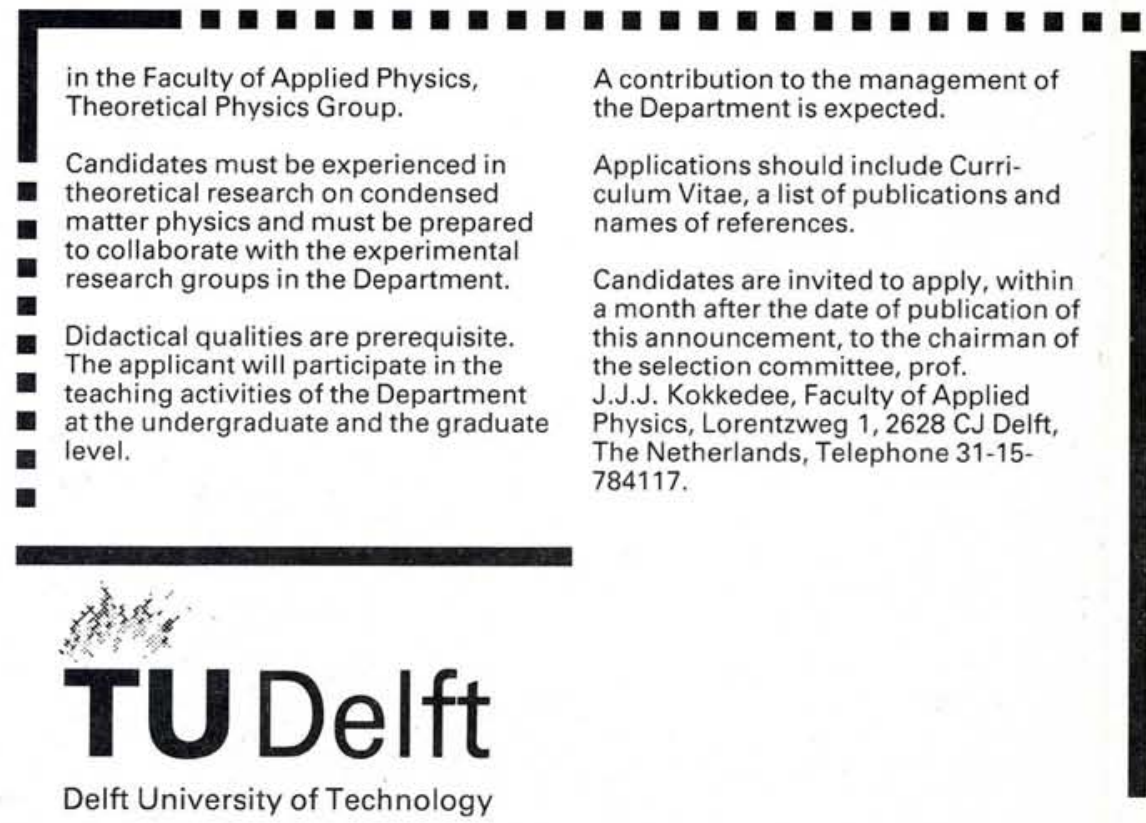

